\title{
Health-related quality of life in Huntington's disease patients: a comparison of proxy assessment and patient self-rating using the disease-specific Huntington's disease health-related quality of life questionnaire (HDQoL)
}

\author{
Mevhibe B. Hocaoglu • E. A. Gaffan • \\ Aileen K. Ho \\ Received: 3 October 2011/ Accepted: 28 December 2011/Published online: 6 March 2012 \\ (C) Springer-Verlag 2012
}

\begin{abstract}
Huntington's disease (HD) is a fatal, neurodegenerative disease for which there is no known cure. Proxy evaluation is relevant for HD as its manifestation might limit the ability of persons to report their health-related quality of life (HrQoL). This study explored patient-proxy ratings of $\mathrm{HrQoL}$ of persons at different stages of $\mathrm{HD}$, and examined factors that may affect proxy ratings. A total of 105 patient-proxy pairs completed the Huntington's disease health-related quality of life questionnaire (HDQoL) and other established HrQoL measures (EQ-5D and SF12v2). Proxy-patient agreement was assessed in terms of absolute level (mean ratings) and intraclass correlation. Proxies' ratings were at a similar level to patients' selfratings on an overall Summary Score and on most of the six Specific Scales of the HDQoL. On the Specific Hopes and Worries Scale, proxies on average rated HrQoL as better than patients' self-ratings, while on both the Specific Cognitive Scale and Specific Physical and Functional Scale proxies tended to rate $\mathrm{HrQoL}$ more poorly than patients themselves. The patient's disease stage and mental wellbeing (SF-12 Mental Component scale) were the two factors that primarily affected proxy assessment. Proxy scores were strongly correlated with patients' self-ratings of HrQoL, on the Summary Scale and all Specific Scales. The patient-proxy correlation was lower for patients at moderate stages of HD compared to patients at early and advanced stages. The proxy report version of the HDQoL is a useful complementary tool to self-assessment, and a promising alternative when individual patients with advanced HD are unable to self-report.
\end{abstract}

M. B. Hocaoglu · E. A. Gaffan · A. K. Ho ( $\square)$

School of Psychology and Clinical Language Sciences,

University of Reading, Reading RG6 6AL, UK

e-mail: aileenkho@ netscape.net
Keywords Huntington's disease - Health-related quality of life · Proxy · Patient-reported outcome · Patient-proxy agreement - Huntington's disease health-related quality of life questionnaire (HDQoL)

Proxy reports have been widely used in health services research [1]. The subjective nature of the concept of healthrelated quality of life (HrQoL) requires its assessment to be carried out by self-report where possible. Proxy reports are relevant when a condition could affect the ability of persons to report or evaluate their subjective states [2,3] such as Huntington's disease (HD). HD is an autosomal-dominant progressively disabling lethal neurodegenerative disorder [4] characterized by a triad of movement disorder, cognitive dysfunction and behavioural disturbances [5]. The progression of HD is viewed in five primary disease stages [6]. At Stage 1 individuals experience slight changes in mood and motor control and remain fully function at home and at work. At moderate stages chorea becomes pronounced, gait is affected and difficulties are experienced with thinking, reasoning, speech and swallowing; individuals may be able to work but at lower capacity (Stage 2) or can no longer work and may need assistance with everyday activities (Stage 3). In advanced stages, HD patients are no longer able to carry out daily activities independently and require the assistance of a carer at home (Stage 4), or need nursing care (Stage 5). Two additional categories are Presymptomatic gene positive (with critical mutation but not yet symptomatic) and At Risk (HD family member but genetic status not yet known). This study considers all seven groups as persons living with $\mathrm{HD}$, since the issue of HrQoL is relevant to all.

In addition to motor impairments [7], cognitive dysfunctions [8], behavioural and psychiatric disturbances 
[9-11] and functional impairments [12], persons living with HD may experience difficulties with insight [13] and may display a lack of awareness [14]. For these reasons, proxy reports in certain instances could be the only point of reference available, as the person might be unable to report their $\mathrm{HrQoL}$ or complete a questionnaire due to various impairments caused by HD.

Studies have attempted to establish patient-proxy agreement in evaluating $\mathrm{HrQoL}$ in other neurodegenerative conditions such as Alzheimer's disease (AD) and Parkinson's disease (PD) [15-17]. Previous research with diseases such as $\mathrm{AD}$ also addressed variability of patient insight across different functional domains $[18,19]$ in order to better understand barriers to self-report [20]. The purpose of the current study was to examine patient and proxy ratings of HrQoL of persons at different stages of HD using the Huntington's disease health-related quality of life questionnaire (HDQoL) and to examine factors that may affect proxy ratings. Proxies separately rated their own $\mathrm{HrQoL}$ using generic instruments, to determine whether a proxy's own health status affects their judgement of a patient's HrQoL.

\section{Methods}

\section{Participants}

Persons living with HD who participated in this study were a subset of a larger validation sample for the HDQoL [21]. All were recruited through a mail-out via the Huntington's Disease Association (HDA) in the UK and provided their consent prior to their inclusion in the study. From this sample, 105 persons living with HD chose to provide proxy data in addition to their own (At risk, $n=10$; Pre-symptomatic gene positive, $n=17$; Stage 1, $n=9$; Stage 2, $n=8$; Stage 3, $n=10$; Stage 4, $n=35$ and Stage 5, $n=15)$. The study was approved by University of Reading Research Ethics Committee and has been performed in accordance with 1964 Declaration of Helsinki.

\section{Procedure and measures}

Research questionnaires were sent to persons living with $\mathrm{HD}$, referred to generically as patients although this includes people who were not yet clinically symptomatic. Those who volunteered to participate were invited to give the accompanying proxy report questionnaires to someone they felt knew them well. Patients were instructed that they might get assistance in completing the questionnaire, from the proxy or another helper, if they found this difficult; however the questionnaire responses should be solely their own. Proxies were instructed to complete the questionnaires independently of the patient, and were asked to focus on how they think the quality of life of the patient has been affected, drawing upon their perceptions and thoughts as a companion/carer. Patients and proxies were requested to return the questionnaires using the pre-paid reply envelope provided within a fortnight.

Patients and proxies were asked to provide demographic details and information regarding patient's functional ability as an indication of disease stage. They were asked to complete three questionnaires as follows.

1. Self- or proxy-report version, respectively, of the HDQoL [21]. This is a disease-specific patient reported outcome measure developed from HD patient interviews, where each item comprises a relevant aspect of health-related quality of life on which HD participants provide a self-reported response on a Likert scale. The proxy-report version is identical to the self-reported patient version, except that proxies are asked their own opinion of the patient for each item. Following exploratory factor analysis and Rasch analysis of data from a larger sample, reported elsewhere [21], several scales can be identified. In the present paper, we report the Summary Scale and the six Specific Scales (i.e. Specific Cognitive, Specific Hopes and Worries, Specific Services, Specific Physical and Functional, Specific Mood State, Specific Self and Vitality) in order that proxy-patient agreement can be assessed both globally and selectively. Each Specific Scale total, and the Summary Scale, ranges from 0 (worst HrQoL) to 100 (best HrQoL).

2. Self-report version of the EQ-5D: note that proxies were here reporting their own health status, not the patient's. This generic HrQoL measure comprises five questions on mobility, self-care, pain, usual activities and psychological status using three-point scales, and generates a Summary Scale $(1=$ Best Health State) and visual analogue scale to indicate general health status (100 = Best Health Status) [22].

3. Self-report version of the SF-12v2: again, proxies reported their own status. This generic measure includes twelve items, measures eight domains of health on five-point scales, and is used to calculate two component scores-the Physical Component Summary Score (PCS) and the Mental Component Summary Score (MCS) [23]. PCS and MCS scales are transformed to $0-100$ scale $(0=$ Worst, $100=$ Best $)$.

Data analyses

All statistical tests are designated significant at $p<0.05$, two-tailed.

Self and proxy agreement on the level of Specific and Summary Scale scores was examined by computing mean 
scores, mean differences and their $95 \%$ confidence intervals. Means were computed for the whole study sample, and for three subgroups according to the disease stage of persons living with HD (Early HD = At risk, Pre-symptomatic gene carriers and Stage 1; Moderate HD = Stages 2 and 3; Advanced HD = Stages 4 and 5). Two-way mixed-design analyses of variance were used to compare the effect of the patient's HD stage subgroup and the type of rater (self or proxy) as independent variables on pairs of self- and proxy-rated Specific and Summary Scale scores (dependent variables).

Multivariate and univariate analyses of covariance were used to examine whether proxy mean scores were accounted for by other potential determinants in addition to patients' HD stage, including the proxy's own HrQoL as reflected in EQ-5D and SF12v2 scores, the patient's EQ$5 \mathrm{D}$ and SFv12 scores, and measures of patient-proxy contact.

To explore agreement in terms of correlation, we examined scatterplots of patient ratings against proxy ratings. Linear regression was used to assess the relationship between self and proxy ratings across the whole sample. Chi-square, Fisher Exact and $t$ tests were used to explore associations between regression standardised residuals (residuals above \pm 1.96 being defined as outliers) and other variables such as HD stage, gender of patients or proxies.

Intraclass correlation coefficients (ICCs) with a one-way random effects model and their respective confidence intervals were calculated to quantify correlation between self and proxy scores. The criteria used were the following: ICC $\geq 0.80$ indicates excellent agreement; $0.80>$ ICC $\geq$ 0.60 substantial agreement; $0.60>\mathrm{ICC} \geq 0.40$ moderate and ICC $<0.40$ poor agreement [16, 24]. ICCs were also calculated separately for patient HD stage subgroups and for cases with Advanced HD who did not receive any assistance in completing the questionnaires.

\section{Results}

Characteristics of the persons living with HD and their proxies are summarised in Table 1. Most of the participants with HD (87\%) had confirmed positive gene status and $67 \%$ had received a clinical diagnosis. Almost all of the proxies were spouses or partners $(83 \%) ; 10 \%$ were parents; $5 \%$ were children of HD individuals and for $2 \%$ of proxies this information was missing. Proxies spent a lot of time per day or night caring for the person living with HD (mean $12.44 \mathrm{~h}$, SD 9.59, range 0:24) and most of them (87\%) had contact on a daily basis. The average time the proxies had known the person living with HD was 30.94 years (SD 15.49, range 1:60). One patient did not report their HD stage, so this patient and proxy were omitted from analyses involving HD stage, giving sample sizes of 36 for Early HD, 18 for Moderate HD and 50 for Advanced HD. The pair was included in whole-sample analyses $(n=105)$. The self-reported EQ-5D and SF-12v2 measures show that, as expected, patients scored worse than proxies on all indices of HrQoL. Proxies' physical health (SF-12v2 PCS) was near the average of available normative data but their mental health (MCS) fell below the population norm $[25,26]$.

Figure 1 shows the patient and proxy ratings separated by patient's HD stage subgroup (Early, Moderate or Advanced).

Two-way mixed-design ANOVAs, rater (self vs. proxy) $\times$ HD stage, yielded strong effects of HD stage on every measure; $F \mathrm{~s}(2,101)$ ranged from 7.47 (Hopes and Worries) to 72.8 (Physical and Functional), $p s \leq 0.001$, with HrQoL ratings becoming progressively poorer across the three groups. There were no significant rater $\times$ HD stage interactions, $F_{\mathrm{S}}(2,101) \leq 2.71$, suggesting that patterns of self and proxy HrQoL scores were broadly consistent across stages. The main effects of rater are reported in Table 2. These were again nonsignificant on the Summary Scale and on all Specific Scales except the Specific Hopes and Worries Scale, where proxy ratings were significantly better than patients' own, and there was a trend for both the Specific Cognitive and the Specific Physical and Functional Scales' proxy ratings to be poorer than self-ratings.

To explore what factors other than patient's HD stage might affect proxies' HDQoL scores, a multivariate analysis of covariance tested the effect of HD stage along with potential covariates on the six Specific Scales and Summary Scale. The covariates were indices obtained from patients' and proxies' EQ-5D and SF-12v2 self-ratings, plus proxy estimates of how long they had known the patient and how much they were in contact. Over and above the expected strong effect of HD stage, the only variable which significantly affected proxy scores (Wilks' lamda $=0.64, p=0.006$ ) was the patient's mental state (as measured by SF-12v2 MCS). Univariate analyses showed that the proxy-rated scales which were affected by patient's MCS were Specific Hopes and Worries, Specific Mood State, Specific Self and Vitality and the Summary Scale-Fs $(1,48) \geq 4.21, p s<0.05$. All slope parameters were positive (0.62-1.72), i.e. the proxy rating of $\mathrm{HrQoL}$ improved as the patient's MCS score improved.

The correlation between proxy and patient HDQoL Summary score ratings is illustrated in Fig. 2 Overall there is a strong linear relationship $\left(\beta=0.81, R^{2}=0.73\right.$, $\mathrm{F}(1,103)=276, p<0.001)$. However outliers (where a proxy's score is greatly discrepant from the patient's own) are evident, commonly in the mid-range of HrQoL and especially among patients with Moderate HD. There were eight regression outliers (cases with standardised residual 
Table 1 Participant demographics and self-reported HrQoL scores, with mean \pm SD and range

$E Q-5 D$ EuroQol questionnaire, $S F-12 v 2$ Short-Form Health Questionnaire, 12-item version 2

\begin{tabular}{lll}
\hline & Persons with HD & Proxies \\
\hline Number of subjects & 105 & 105 \\
Female/male & $61 / 44$ & $55 / 50$ \\
Age & $56.42 \pm 12.82(17: 90)$ & $56.55 \pm 12.89(17: 90)$ \\
Full-time education (years) & $13.77 \pm 4.50(4: 35)$ & $13.25 \pm 4.07(0: 25)$ \\
Full or part-time employment/ & $20 / 85$ & $49 / 56$ \\
$\quad$ retired or unemployed & $58.38 \pm 23.20(0: 100)$ & $75.31 \pm 20.31(0: 100)$ \\
EQ-5D Visual Analogue Scale (VAS) & $0.56 \pm \mathrm{SD}=0.35(-0.33: 1)$ & $0.78 \pm 0.22(-0.04: 1)$ \\
EQ-5D Index Score & $41.52 \pm 12.78(16.33: 66.20)$ & $50.67 \pm 8.94(30.05: 64.73)$ \\
SF-12v2 Physical Component & & \\
$\quad$ Score (PCS) & $39.95 \pm 11.50(13.89: 67.34)$ & $43.85 \pm 11.25(11.83: 70.29)$ \\
SF-12v2 Mental Component & & \\
$\quad$ Score (MCS) & &
\end{tabular}
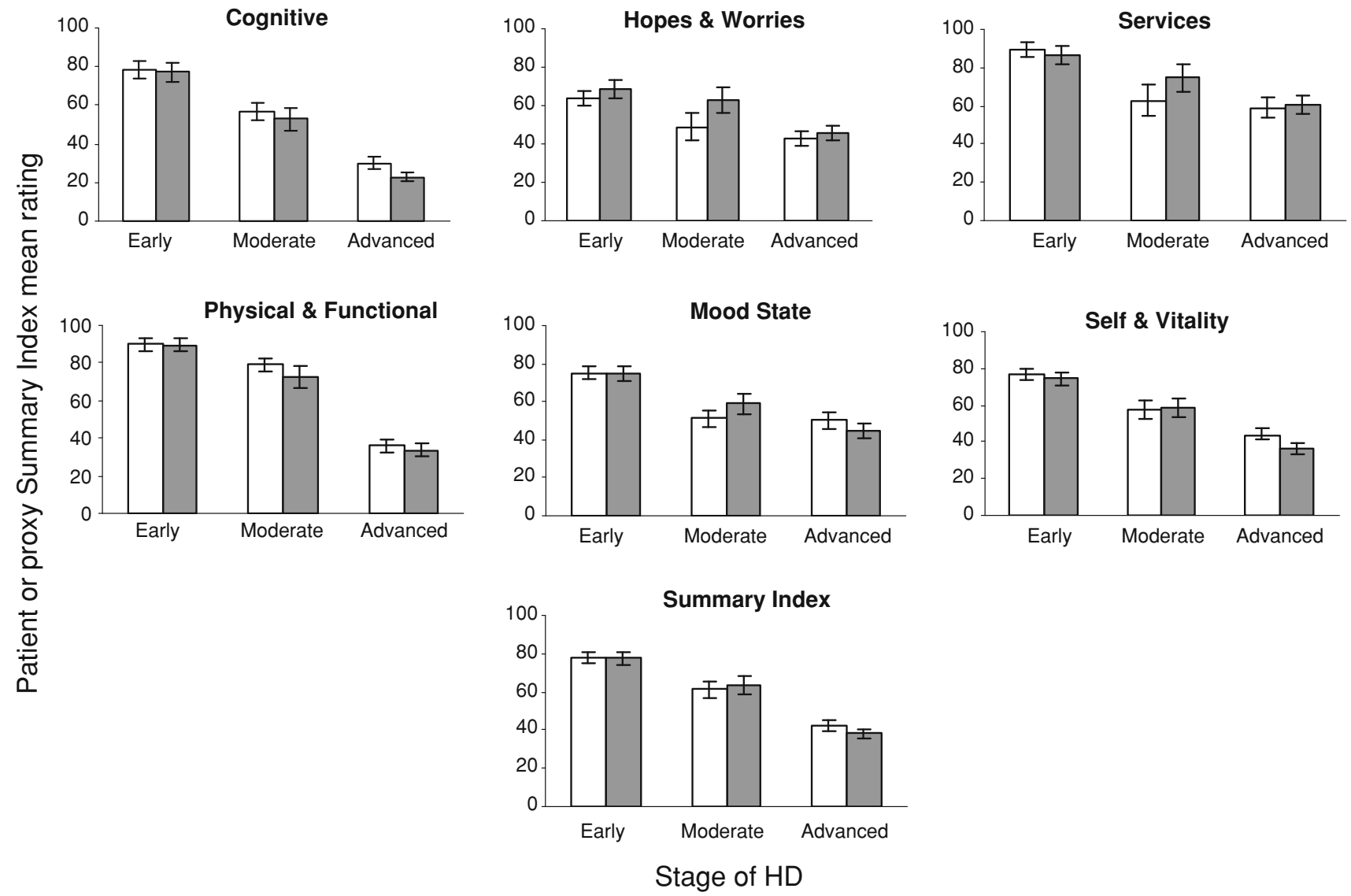

Fig. 1 Mean ratings by patients (white bars) and proxies (dark bars) \pm SE, on the six Specific Scales and Summary Score of the HDQoL. Means are shown separately for Early stage HD $(n=36)$, Moderate stage HD $(n=18)$ and Advanced stage HD patients $(n=50)$

greater than \pm 1.96 ). One outlier pair (patient score of 27 and proxy score of 59) was the one whose patient did not report their HD stage (and is therefore missing from the plot). Among the remaining seven outlier pairs, two patients were Early stage, four Moderate and one Advanced. Thus, outliers were significantly more likely to come from the Moderate HD subgroup than the other subgroups, Fisher Exact $p=0.016$. Information supplied by the proxy implies that the patient in the eighth pair, missing from the analysis, also probably had Moderate HD.

We explored other potential associates, including gender of patient and proxy, nature of proxy-patient relationship, proxy's own HrQoL and frequency of contact. None were significant, and there was only a trend for proxy gender, Fisher Exact $p=0.061$; seven out of eight outlier proxies were male compared to 43 out of 97 others. This effect is 
Table 2 Mean HDQoL Specific and Summary Scale scores by rater

\begin{tabular}{|c|c|c|c|c|c|c|}
\hline \multirow[t]{2}{*}{$\mathrm{HDQOL}^{\mathrm{a}}$} & \multirow{2}{*}{$\begin{array}{l}\text { Self-rated }(n=105) \\
\text { Mean }(\mathrm{SD})\end{array}$} & \multicolumn{3}{|c|}{ Proxy-rated $(n=105)$} & \multicolumn{2}{|c|}{ ANOVA rater effect ${ }^{c}$} \\
\hline & & Mean (SD) & Mean $\operatorname{diff}^{b}$ & $95 \% \mathrm{CI}$ & $F(1,101)$ & $p$ \\
\hline \multicolumn{7}{|l|}{ Specific Scales } \\
\hline Cognitive & $51.34(32.24)$ & $46.52(33.98)$ & 4.82 & 0.74 to 8.90 & 3.45 & 0.07 \\
\hline Hopes and Worries & $50.98(27.93)$ & $56.89(30.57)$ & -5.90 & -9.90 to -1.91 & 11.32 & 0.001 \\
\hline Services & $69.71(34.57)$ & 72.37 (33.97) & -2.65 & -7.74 to 2.43 & 1.75 & ns \\
\hline Physical and Functional & $62.05(33.56)$ & $59.97(34.80)$ & 2.08 & -1.08 to 5.25 & 2.90 & 0.09 \\
\hline Mood State & $58.66(28.08)$ & $57.42(28.12)$ & 1.24 & -2.77 to 5.24 & $<1$ & ns \\
\hline Self and Vitality & $57.39(25.78)$ & $53.60(26.75)$ & 3.78 & 0.21 to 7.35 & 2.22 & ns \\
\hline Summary Scale & $57.71(25.03)$ & $56.2(26.46)$ & 1.50 & -1.21 to 4.21 & $<1$ & $\mathrm{~ns}$ \\
\hline
\end{tabular}

$C I$ confidence interval, $H D Q o L$ Huntington's disease health-related quality of life questionnaire

${ }^{\text {a }}$ HDQOL scales are scored on a 0 (worst HrQoL) - 100 (best HrQoL) scale

b A positive mean difference indicates that self-rated scale score is higher than proxy-rated scale score

${ }^{c}$ Effect of rater (self vs. proxy) in 2-way mixed ANOVA, rater $\times$ HD Stage

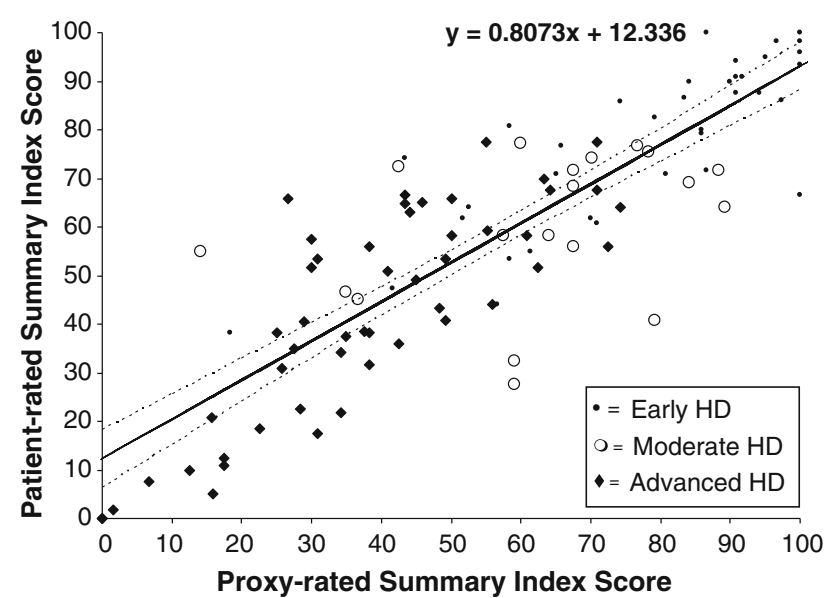

Fig. 2 Plot of patient-rated versus proxy-rated Summary Scale scores, with regression line and $95 \%$ CIs based on all data. Symbols represent patient's stage of HD: bullet Early, shadowed white circle Moderate, filled diamond Advanced

independent from the preceding one, because Moderate subgroup proxies were not more likely to be male than those in other subgroups, $\chi^{2}<1$. The excess of male proxies among outliers is not simply an extreme case of generally poor prediction of patient HrQoL by males; after removing all outliers, the absolute standardised residuals of male proxies were similar to those of females (male $\mathrm{M}=0.68$, female $\mathrm{M}=0.65, \mathrm{t}(95)<1)$.

Scatterplots of other subscales (not shown) show the same pattern of lower proxy-patient agreement and more outliers in the moderate HD group. This is confirmed by the ICCs, see Table 3. ICC values for the whole sample reflected excellent $(>0.80)$ or substantial $(>0.60)$ agreement on all scales, as did virtually all values for the Early and Advanced HD subgroups separately. In the Moderate HD subgroup, agreement was moderate $(<0.60)$ to poor $(<0.40)$.
Agreement in the Advanced HD subgroup might have been inflated because Advanced HD patients were more likely than others to have reported that they received assistance from proxies in completing the questionnaires, $\left(\chi^{2}(2, N=104)=15.56, \quad p<0.001\right)$. Therefore, ICCs were recomputed for the Advanced HD subgroup after removing the 16 cases (out of a total of 50) who received assistance. ICCs for this subset were slightly lower than for the whole Advanced subgroup-e.g. Summary Scale ICC $=0.71$ compared to 0.81 for the whole subgroup - but agreement remained substantial on all scales except for Cognitive, and Self and Vitality, where it was moderate though still highly significant.

\section{Discussion}

Previous reports on the carers of patients with HD have examined their reports of their own HrQoL and compared it to that of patients [27-29]. However, the current study using the proxy version of the HDQoL is the first in which carers or others act as proxies, providing their own perceptions of the HrQoL of persons living with HD, maintaining a 'proxy-proxy' perspective [30].

Individual level correlations and group level analyses showed that patient-proxy agreement was substantial to excellent for the Summary Scale score of the HDQoL, and also for most of its Specific Scales. On the Specific Hopes and Worries Scale, proxies rated patients' HrQoL as better than patients' own ratings, while on both the Specific Cognitive Scale and Specific Physical and Functional Scale the reverse was true. The literature shows that for chronically ill patients including those with neurological disorders such as stroke [31], brain cancer [32] and Parkinson's disease $[15,16]$, patient-proxy agreement on validated 
Table 3 Intraclass correlation coefficients between self and proxy scores for the HDQoL Specific and Summary Scales: whole sample and HD stage subgroups

\begin{tabular}{|c|c|c|c|c|c|c|c|c|}
\hline \multirow[t]{2}{*}{ HDQoL } & \multicolumn{2}{|c|}{ Whole sample $(n=105)$} & \multicolumn{2}{|c|}{ Early HD $(n=36)$} & \multicolumn{2}{|c|}{ Moderate HD $(n=18)$} & \multicolumn{2}{|c|}{ Advanced HD $(n=50)$} \\
\hline & ICC & $(95 \% \mathrm{CI})$ & ICC & $(95 \% \mathrm{CI})$ & ICC & $(95 \% \mathrm{CI})$ & ICC & $(95 \% \mathrm{CI})$ \\
\hline \multicolumn{9}{|l|}{ Specific Scales } \\
\hline Cognitive & 0.79 & $(0.71-0.85)$ & 0.78 & $(0.61-0.88)$ & -0.03 & $(-0.47$ to 0.42$)$ & 0.61 & $(0.40-0.76)$ \\
\hline Hopes and Worries & 0.74 & $(0.63-0.81)$ & 0.83 & $(0.69-0.91)$ & 0.49 & (0.05 to 0.77$)$ & 0.77 & $(0.63-0.86)$ \\
\hline Services & 0.71 & $(0.60-0.79)$ & 0.76 & $(0.58-0.87)$ & 0.48 & (0.04 to 0.76$)$ & 0.74 & $(0.59-0.85)$ \\
\hline Physical and Functional & 0.88 & $(0.84-0.92)$ & 0.87 & $(0.77-0.93)$ & 0.24 & $(-0.23$ to 0.63$)$ & 0.81 & $(0.69-0.89)$ \\
\hline Mood State & 0.73 & $(0.63-0.81)$ & 0.56 & $(0.29-0.75)$ & 0.46 & $(0.02$ to 0.76$)$ & 0.76 & $(0.61-0.85)$ \\
\hline Self and Vitality & 0.75 & $(0.65-0.82)$ & 0.63 & $(0.39-0.79)$ & 0.65 & $(-0.28$ to 0.85$)$ & 0.59 & $(0.37-0.74)$ \\
\hline Summary Scale & 0.85 & $(0.79-0.90)$ & 0.81 & $(0.65-0.90)$ & 0.37 & ( -0.09 to 0.70$)$ & 0.81 & $(0.69-0.89)$ \\
\hline
\end{tabular}

$H D$ Huntington's disease, ICC intraclass correlation coefficient, $C I$ confidence interval, HDQoL Huntington's disease health-related quality of life questionnaire

disease-specific questionnaires are generally moderate to high, and when discrepant, proxies often provide poorer QoL ratings than patients [30] [33]. Our data are consistent with the literature, but it is notable that proxies rated patients' Specific Hope and Worries Scale score as better rather than worse than patients' own ratings. This dimension may be particularly difficult for proxies to gauge as it is perhaps even more internal than other psychosocial dimensions, which can garner lower agreement than more objective physical dimensions [32].

When patient-proxy agreement is examined across disease severity, the Early and Advanced subgroups showed substantial to excellent agreement on the Summary Score as well as for the Specific Scale scores. In both subgroups, the highest ICC values emerged on the most 'objective', observable scale-Specific Physical and Functional-as has often been reported elsewhere [33]. Yet even the more 'subjective' scales such as Specific Hopes and Worries, or Specific Mood State, yielded substantial ICCs. This may reflect the fact that proxies were long-term companions or close family members who typically show better agreement than unrelated healthcare providers [17, 34]. The HDQoL showed good patient-proxy agreement, not only with early HD patients who could validly assess their own HrQoL, but also with Advanced HD patients who usually have physical or cognitive barriers to self-reporting. Good proxy-patient agreement in the Advanced group was not merely an artefact of proxies assisting with questionnaire completion, because agreement remained substantial even after excluding patients who received assistance. This is in contrast to poorer agreement reported for more advanced stage Alzheimer's and Parkinson's disease patients [15-17]. To confirm these postal study results, further investigation where questionnaire completion is fully monitored would be useful.

For moderate Stage 2 and 3 patients, patient-proxy agreement was moderate to poor. The QoL cancer severity literature reports a similar pattern of results where larger patient-proxy discrepancies were more frequent for patients who were slightly and moderately affected [33], rather than at extreme ends of the spectrum. This may be due to greater scope for variability in ratings of patients and proxies at an intermediate level of disease manifestation. Progression of HD is suggested to be fastest, more variable and diverse in the moderate stages, compared to early or advanced stages of the disease [35]. Therefore, patients with moderate HD might be the most heterogeneous group and most changeable over time, making it hard for proxies to form stable judgements. Furthermore, the moderate stages may be particularly complex for patients, who are beginning to experience a physical and psychological decline which both they and their family have long dreaded. They may be anxious about other people's attitudes, or wrongly believe that transient difficulties are signs of HD, as we have previously found [36]. Some proxies may find it hard to communicate with patients and understand their subtle and fluctuating symptoms at this stage, and our analysis hints that this may be particularly true of male proxies. By comparison, advanced-stage patients' state and relations with carers are more stable, perhaps making it easier for proxies to evaluate HrQoL. It is also worth noting that the 'proxy-proxy' perspective adopted here-where proxies were encouraged to use their own viewpoint, rather than explicitly trying to 'simulate' the patient's feelingscan lead to genuine differences between proxy and patient views of HrQoL [30].

We examined factors that might influence proxies' tendency to over- or under-rate HrQoL. Previous studies have suggested that proxies' level of burden, psychosocial stress and amount of time they spend with patients could moderate their assessment [1, 37-39]. In the present case, neither proxies' own physical or psychosocial HrQoL (as putative measures of carer burden), nor their degree of 
contact with the patient, influenced the level of proxy ratings. However, the patient's own psychological state (SF-12v2 Mental Component score) influenced proxy scores on the more 'subjective' Specific Scales of the HDQoL-Specific Hopes and Worries, Specific Mood State, Specific Self and Vitality-even after the patient's disease stage was taken into account. Therefore, proxies' ratings of psychosocial aspects of $\mathrm{HrQoL}$ were sensitive to patients' psychological state, as well as symptom severity.

In summary, this cross-sectional study addressed parameters of patient-proxy agreement on HrQoL for the first time in HD, by comparing proxy assessment with patient self-report, using the disease-specific HDQoL. There was substantial agreement for the Summary Scale and also the Specific Scales and this suggests that the HDQoL proxy version yields generally similar scores to patients' own, particularly on physical and functional aspects but also on more 'subjective' scales. On the Specific Hopes and Worries Scale, proxies on average rated HrQoL as better than patients' self-ratings, while on both the Specific Cognitive and Specific Physical and Functional Scales proxies tended to rate $\mathrm{HrQoL}$ more poorly than patients themselves. As more modest patient-proxy agreement was found for moderate-stage patients, proxy report should not be used as a substitute for patient-reported rating for these patients. As there was good patient-proxy agreement for advanced-stage patients, proxy evaluation using this questionnaire could be a particularly useful complementary tool to self-report in advanced HD.

Acknowledgments This study was funded by a Grant from the European Huntington's disease Network to Aileen K Ho. The authors would like to thank all of the study participants and their family members who participated in the project and HDA UK for their kind support. The authors would also like to thank the European Huntington's Disease Network by the Quality of Life Working Group: Walter Bucher, Alyson Bradbury, Beatrice de Schepper, John Eden/ Sue Beevers and team, George El-Nimr, Victor Hendrikx, Alis Hughes, Diana King, Ursula Kleibrink, Rita Kuttruff-Wilschut, Bernhard Landwehermeyer, Anne Lenon-Bird/Catherine Paradise and team, Christiane Lohkamp, Rhona MacLeod, Helene Padieu, MarieOdile Perrousseaux, Asuncion Martinez, Lilliane Rapaille, Helen Santini, Pavla Sasinkova, Beverley Soltysiak, Steve Smith, Hans van der Leer, Lucienne van der Meer, Jo Anne Watton and team, Marleen van Walsem, Michael Wooldridge, Paola Zinzi.

Conflicts of interest The authors declare that they have no conflict of interest.

\section{References}

1. Snow LA, Cook KF, Lin PS, Morgan RO, Magaziner J (2005) Proxies and other external raters: methodological considerations. Health Serv Res 40(5 Pt 2):1676-1693

2. Sainfort F, Becker M, Diamond R (1996) Judgments of quality of life of individuals with severe mental disorders: patient self-report versus provider perspectives. Am J Psychiatr 153(4): 497-502

3. Albert SM, Del Castillo-Castaneda C, Sano M, Jacobs DM, Marder K, Bell K et al (1996) Quality of life in patients with Alzheimer's disease as reported by patient proxies. J Am Geriatr Soc 44(11):1342-1347

4. Novak MJU, Tabrizi SJ (2010) Huntington's disease. BMJ 340(jun30_4):c3109

5. Biglan K, Shoulson I (2002) Huntington's disease. In: Jankovic J, Tolosa E (eds) Parkinson's disease and movement disorders. Lipincott Williams and Wilkins, Philedelphia, pp 298-378

6. Shoulson IF, Fahn S (1979) Huntington disease: clinical care and evaluation. Neurology 29(1):1-3

7. Phillips JG, Bradshaw JL, Chiu E, Teasdale N, Iansek R, Bradshaw JA (1996) Bradykinesia and movement precision in Huntington's disease. Neuropsychologia 34(12):1241-1245

8. Lemiere J, Decruyenaere M, Evers-Kiebooms G, Vandenbussche E, Dom R (2004) Cognitive changes in patients with Huntington's disease (HD) and asymptomatic carriers of the HD mutation. J Neurol 251(8):935-942

9. Kingma EM, van Duijn E, Timman R, van der Mast RC, Roos RAC (2008) Behavioural problems in Huntington's disease using the Problem Behaviours Assessment. Gen Hosp Psychiatr 30(2): $155-161$

10. Craufurd D, Thompson J, Snowden J (2001) Behavioral changes in Huntington disease. Neuropsychiatr Neuropsychol Behav Neurol 14(4):219-226

11. Paulsen JS, Nehl C, Hoth KF, Kanz JE, Benjamin M, Conybeare $\mathrm{R}$ et al (2005) Depression and stages of Huntington's disease. J Neuropsychiatr Clin Neurosci 17(4):496-502

12. Rothlind JC, Bylsma FW, Peyser C, Folstein SE, Brandt J (1993) Cognitive and motor correlates of everyday functioning in early Huntington's disease. J Nerv Ment Dis 181(3):194-199

13. Ho AK, Robbins AOG, Barker RA (2006) Huntington's disease patients have selective problems with insight. Mov Disord 21(3):385-389

14. Duff K, Paulsen JS, Beglinger LJ, Langbehn DR, Wang C, Stout JC et al (2010) "Frontal" behaviors before the diagnosis of Huntington's disease and their relationship to markers of disease progression: evidence of early lack of awareness. J Neuropsychiatr Clin Neurosci 22(2):196-207

15. Martínez-Martín P, Benito-León J, Alonso F, Catalán MJ, Pondal M, Zamarbide I (2004) Health-related quality of life evaluation by proxy in Parkinson's disease: approach using PDQ-8 and EuroQoL-5D. Mov Disord 19(3):312-318

16. Fleming A, Cook KF, Nelson ND, Lai EC (2005) Proxy reports in Parkinson's disease: caregiver and patient self-reports of quality of life and physical activity. Mov Disord 20(11):1462-1468

17. Novella JL, Jochum C, Jolly D, Morrone I, Ankri J, Bureau F et al (2001) Agreement between patients' and proxies' reports of quality of lifein Alzheimer's disease. Qual Life Res 10(5):443452

18. Green J, Goldstein FC, Sirockman BE, Green RC (1993) Variable awareness of deficits in Alzheimer's disease. Cogn Behav Neurol 6(3):159-165

19. Vasterling JJ, Seltzer B, Foss MW, Vanderbrook V (1995) Unawareness of deficit in Alzheimer's disease: domain-specific differences and disease correlates. Cogn Behav Neurol 8(1):2632

20. Trigg R, Jones RW, Skevington SM (2007) Can people with mild to moderate dementia provide reliable answers about their quality of life? Age Ageing 36(6):663-669

21. Hocaoglu MB, Gaffan EA, Ho AK (2012) The Huntington's disease health-related quality of life questionnaire (HDQoL): a disease-specific measure of health-related quality of life. Clin Genet 81(2):117-122 
22. EuroQol Group (1990) EuroQol-a new facility for the measurement of health-related quality of life. Health Policy 20(3):321-328

23. Ware J, Kosinski M, Turner-Bowker D, Gandek B (2002) SF12v2: how to score version 2 of the SF-12 health survey. QualityMetric Incorporated, Lincoln

24. Landis JR, Koch GG (1977) The measurement of observer agreement for categorical data. Biometrics 33(1):159-174

25. Kind P, Dolan P, Gudex C, Williams A (1998) Variations in population health status: results from a United Kingdom national questionnaire survey. BMJ 316(7133):736-741

26. Ware JE Jr, Kosinski M, Turner-Bowker DM, Gandek B (2002) User's manual for the SF-12v2 ${ }^{\circledR}$ health survey with a supplement documenting SF-12 ${ }^{\circledR}$ health survey. QualityMetric Incorporated, Lincoln

27. Aubeeluck A, Buchanan H (2007) The Huntington's disease quality of life battery for carers: reliability and validity. Clin Genet 71(5):434-445

28. Kaptein AA, Scharloo M, Helder DI, Snoei L, van Kempen GM, Weinman J et al (2007) Quality of life in couples living with Huntington's disease: the role of patients' and partners' illness perceptions. Qual Life Res 16(5):793-801

29. McCabe MP, Firth L, O'Connor E (2009) A comparison of mood and quality of life among people with progressive neurological illnesses and their caregivers. J Clin Psychol Med Settings 16(4):355-362

30. Pickard AS, Knight SJ (2005) Proxy evaluation of health-related quality of life: a conceptual framework for understanding multiple proxy perspectives. Med Care 43(5):493-499
31. Hilari K, Owen S, Farrelly SJ (2007) Proxy and self-report agreement on the stroke and aphasia quality of life scale-39. J Neurol Neurosurg Psychiatr 78(10):1072-1075

32. Sneeuw KCA, Aaronson NK, Osoba D, Muller MJ, Hsu M-A, Yung A et al (1997) The use of significant others as proxy raters of the quality of life of patients with brain cancer. Med Care 35(5):490-506

33. Sneeuw KC, Sprangers MA, Aaronson NK (2002) The role of health care providers and significant others in evaluating the quality of life of patients with chronic disease. J Clin Epidemiol 55(11):1130-1143

34. Andresen EM, Vahle VJ, Lollar D (2001) Proxy reliability: health-related quality of life (HrQoL) measures for people with disability. Qual Life Res 10(7):609-619

35. Mahant N, McCusker EA, Byth K, Graham S (2003) Huntington's disease: clinical correlates of disability and progression. Neurology 61(8):1085-1092

36. Ho AK, Hocaoglu MB, European Huntington's Disease Network Quality of Life Working Group (2011) Impact of Huntington disease across the entire disease spectrum: the phases and stages of disease from the patient perspective. Clin Genet 80(3):235-239

37. Rothman ML, Hedrick SC, Bulcroft KA, Hickam DH, Rubenstein LZ (1991) The validity of proxy-generated scores as measures of patient health status. Med Care 29(2):115-124

38. Magaziner J (1997) Use of proxies to measure health and functional outcomes in effectiveness research in persons with Alzheimer disease and related disorders. Alzheimer Dis Assoc Disord 11(Suppl 6):168-174

39. von Essen L (2004) Proxy ratings of patient quality of life. Acta Oncol 43(3):229-234 